\title{
Article \\ Evaluation of Acoustic Waves in Acousto-Optical Devices by Ultrasonic Imaging
}

\author{
Sergey A. Titov *, Alexander S. Machikhin and Vitold Ed. Pozhar
}

check for updates

Citation: Titov, S.A.; Machikhin, A.S.; Pozhar, V.E. Evaluation of Acoustic

Waves in Acousto-Optical Devices by

Ultrasonic Imaging. Materials 2022,

15, 1792. https://doi.org/10.3390/

ma15051792

Academic Editor: Alexander N.

Obraztsov

Received: 31 December 2021

Accepted: 20 February 2022

Published: 27 February 2022

Publisher's Note: MDPI stays neutral with regard to jurisdictional claims in published maps and institutional affiliations.

Copyright: (C) 2022 by the authors. Licensee MDPI, Basel, Switzerland. This article is an open access article distributed under the terms and conditions of the Creative Commons Attribution (CC BY) license (https:// creativecommons.org/licenses/by/ $4.0 /)$.
Scientific and Technological Center of Unique Instrumentation, Russian Academy of Sciences, 15 Butlerova, 117342 Moscow, Russia; np@ntcup.ru (A.S.M.); vitold@ntcup.ru (V.E.P.)

* Correspondence: titov.sa@ntcup.ru; Tel.: +7-(495)333-24-31

\begin{abstract}
The structure of the acoustic field defines the key parameters of acousto-optical (AO) devices. To confirm their compliance with the expected values in the presence of multiple real factors, AO crystalline cells require accurate experimental investigation of the acoustic field after being totally assembled. For this purpose, we propose to detect and quantify all the acoustic waves propagating in AO cells using an impulse acoustic microscopy technique. To validate this approach, we have analyzed both theoretically and experimentally the modes, amplitudes, propagation trajectories, and other features of the ultrasonic waves generated inside an AO modulator made of fused quartz. Good correspondence between theoretical and experimental data confirms the effectiveness of the proposed technique.
\end{abstract}

Keywords: acousto-optic crystals; propagation of acoustic waves; non-destructive testing; impulse acoustic microscopy

\section{Introduction}

Acousto-optic (AO) interaction is a physical effect, which is used for modulation, deflection, and spectral and spatial filtration of electromagnetic radiation $[1,2]$. As they are compact, monolithic and free of moving components, AO devices are now widespread in industrial, biomedical and scientific applications [3-5]. The physical principle of most $\mathrm{AO}$ instruments consists of Bragg diffraction of light by ultrasound in crystalline media. The tuning of ultrasound power and frequency enables smooth and accurate control of amplitude, propagation direction, polarization and other parameters of light waves.

The structure of induced acoustic fields defines the performance of AO devices to a large extent. Attenuation, divergence, walk-off and other features of ultrasonic waves inevitably influence the key characteristics of AO diffraction: efficiency, signal-to-noise ratio, light beam distortions, etc. Precise theoretical consideration and modeling give a chance to predict and protect the characteristic degradation at the design stage, but the real structure of an acoustic field may only be revealed experimentally. It is barely possible to reveal and quantify crystal inhomogeneity and inner defects, heat generation by the piezoelectric transducer, multiple reflections of ultrasound from AO cell facets, and many other factors without experiments [6-8]. Therefore, it is essential to carefully examine each assembled AO cell and certify it in terms of the real acoustic field structure.

The Schlieren method has been adopted as a standard optical technique for imaging acoustic fields in homogeneous transparent media including AO crystals $[1,9,10]$ since it provides a two-dimensional projection image formed due to the diffraction of light on an acoustic beam.

In the interferometric [11,12] and holographic [13] techniques, the spatio-temporal distribution of the probing wide laser beam is recorded by a digital camera. Since the output signal changes at the frequency of the sonic radiator, these techniques are not applicable to high-frequency AO devices. Laser beam scanning also enables visualization of acoustic fields [14-17]. In [14-16], the laser beam diameter is smaller than the sound wavelength; 
therefore, this scheme is applicable only to low-frequency fields. In [17], the shape of the diffracted laser beam allows evaluation of high frequency ultrasonic wave in solid media.

In all these methods [9-17], the measurement results are in fact averaged over the entire optical path through the sound field. Therefore, in practice, they are effective for the analysisof simple sound fields that have plane or symmetrical wave fronts. In real $\mathrm{AO}$ devices, many waves of various types with different directions and amplitudes can propagate. To obtain three-dimensional distributions of ultrasonic fields, tomographic methods based on the interferometric [18-20] or advanced Schlieren schemes [21-23] are applicable. In this case, data acquisition from different angles is necessary, which is hardly possible for $\mathrm{AO}$ devices due to their design features.

The acoustic field structure in crystal influences the wave displacement distribution on the facet of an AO cell located opposite or next to the transducer. Such measurement is available for the laser ultrasonic technique [24] but needs a highly reflective facet, which is normally absent. The gold standard for measuring the acoustic field is the use of needle hydrophones $[25,26]$. However, they cannot be placed directly on the AO cell facet and have low sensitivity due to their small dimensions. In this paper, we propose to use a focused immersion ultrasonic transducer with the focus placed on the cell surface. This scheme is typical for the receiver of a pulse acoustic scanning microscope [27], which has a high signal-to-noise ratio and spatio-temporal resolution.

In this study, we intend to validate this approach by studying an $\mathrm{AO}$ modulator made of fused quartz in order to determine both theoretically and experimentally the modes, amplitudes, propagation trajectories and other features of the ultrasonic waves inside. Below, we describe the experimental setup, the theoretical model of acoustic field structure and the experimental data, then discuss the results and demonstrate good correspondence between theoretical prediction and acoustic microscopy data that confirm the effectiveness of the proposed technique.

\section{Experimental Setup and Technique}

To confirm the feasibility of this approach, we applied it to testing an AO cell 1 made of optical fused quartz (Figure 1). It has the shape of a straight prism with a thickness of $12 \mathrm{~mm}$. To generate the longitudinal acoustic waves in the cell, a lithium niobate ultrasonic transducer 2 is attached to the bottom plane. To avoid the formation of standing acoustic waves, the upper plane of the cell is inclined at the angle $\alpha=6.5^{\circ}$. The transducer consists of two $17.5 \times 3.5 \mathrm{~mm}^{2}$ sections separated by a $4 \mathrm{~mm}$ gap. The central frequency is $50 \mathrm{MHz}$ while the frequency range is $30 \mathrm{MHz}$.

The upper part of the cell is inserted into a cuvette 3 filled with an immersion liquid (water). The ultrasonic waves generated by the piezoelectrical transducer 2 propagate inside the $\mathrm{AO}$ cell 1 and partially penetrate into the immersion liquid. These waves are detected by a focused piezotransducer 4 with a central frequency of $50 \mathrm{MHz}$ and an angular aperture of $15^{\circ}$. The focus of the transducer 4 located at the quartz-water interface is mechanically translated along this plane to record the spatial distribution of the waves.

The ultrasonic data acquisition system of the experimental setup consists of the blocks typical for a scanning acoustic microscope $[27,28]$. To separate responses of different waves, the pulsed mode is used. The pulser-receiver 5 (5073PR, Panametrics-NDT Inc., Waltham, MA , USA) generates short electrical pulses to feed the AO piezotransducer 2 and amplifies the weak output signals of the transducer 4 . The signals are then processed by the analog-to-digital converter 6 (FM412x500M, Insys Inc., Moscow, Russia) at a sampling rate of $500 \mathrm{MHz}$ and a resolution of 12 bits. The mechanical movement of the focused transducer is implemented by the motorized translation stage 7 (8MT167-100, Standa Ltd., Vilnius, Lithuania). At each position of the transducer, the acquired signal is averaged over 16 series in order to increase the signal-to-noise ratio and then is recorded as a function of the wave propagation time $t$ within temporal widows of $4 \mu \mathrm{s}$. We acquired the signals at different positions separated by a spatial period of $0.1 \mathrm{~mm}$. The travel distance is set to $44 \mathrm{~mm}$ to cover the entire top facet of the AO cell. Recording time of one scan is about $4 \mathrm{~s}$. 
To obtain the delayed multiple reflection responses, the scan is repeated several times with different time window settings and signal gain.

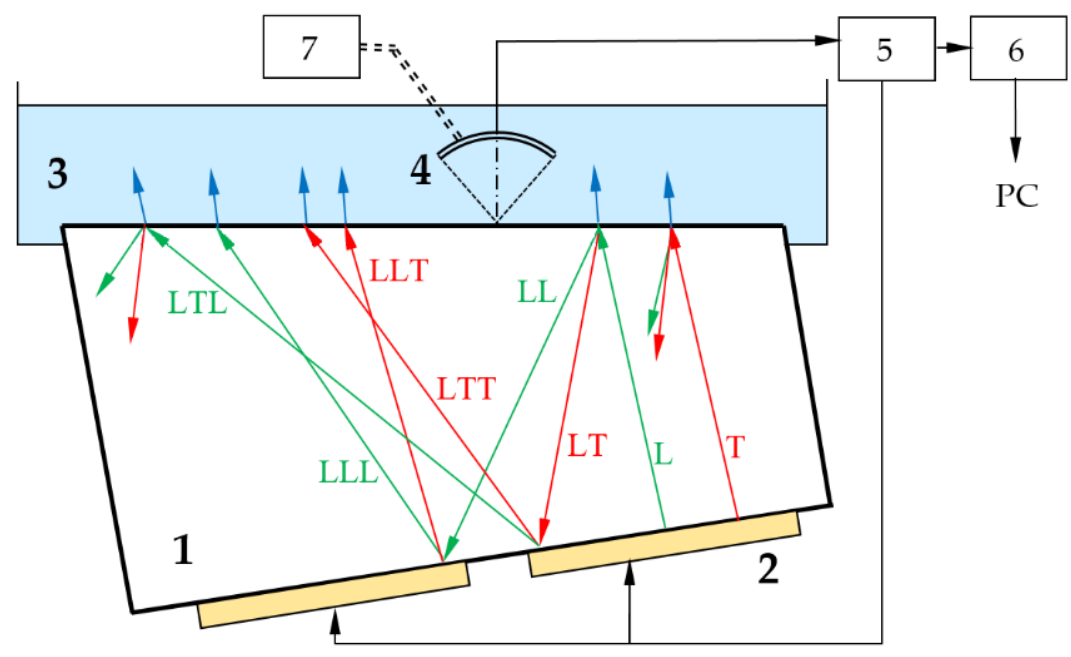

(a)

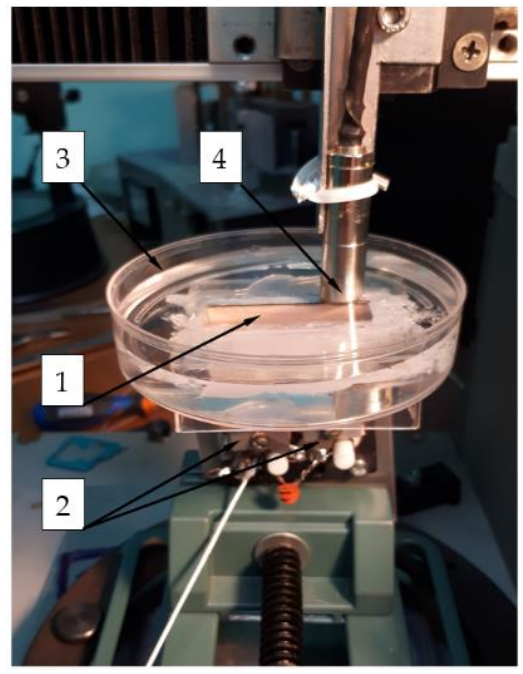

(b)

Figure 1. Scheme (a) and appearance (b) of the experimental setup: 1 -AO cell; 2 -emitting piezotransducer; 3-cuvette with immersion liquid; 4-receiving piezotransducer; 5-pulser-receiver; 6-analog-to-digital converter; 7-motorized translator. Green and red arrows show longitudinal $(L)$ and transverse $(T)$ waves propagating in AO cell, blue arrows correspond to longitudinal waves in liquid.

There is a variety of ultrasonic waves propagating inside the AO cell (Figure 1), both longitudinal and transverse, while in the immersion liquid there are only longitudinal ones. Normally, the transducer generates a plane longitudinal wave $L$, but a weak transverse wave $T$ may also appear. Both waves undergo reflection and mode conversion at the upper solidliquid interface and partially penetrate the water. Wave $L$ produces a longitudinal wave LL and a transverse wave LT, which transform at the bottom plane into two longitudinal waves, LLL and LTL, and two transverse waves, LLT and LTT.

The applied technique presumes detection of the water-penetrating waves by a scanning transducer focused on the upper face of the AO cell. These signals, together with information about the acoustic properties of the cell and immersion liquid are necessary to calculate the amplitudes of upward and downward waves in the AO cell. Then, we may evaluate the amplitudes of the waves inside the $\mathrm{AO}$ device using the characteristics of the acoustic absorber. To verify the theoretical data, we detect the waves on the clear part of the upper face partly covered with the absorber.

\section{Theoretical Model}

In the theoretical analysis described below, we have made the following assumptions. Since the dimensions of the transmitting transducer are much larger than the ultrasound wavelength, the divergence is rather small, the wave fronts in quartz and water are approximately plane and the ray approximation is applicable. As the acoustic attenuation in quartz is small, the amplitudes in the bottom face and near the quartz-water interface are equal. Therefore, the well-known formulas for the reflection, transmission and mode conversion coefficients for plane waves at the solid-liquid and solid-solid interfaces are valid [29]. All the coefficients are real because the angles of incidence do not exceed the critical values. The angles between the wave vectors in water and the axis of the receiving transducer are significantly less than its angular aperture. The distance between the transducer and the interface is constant and, therefore, the sensitivity of the receiver does not depend on the wave propagation direction. 
In theoretical analysis, we use the symbols $\eta, \mu, \xi, \kappa=L$, and $T$ to denote waves. Let $p_{\eta}$ be the amplitudes of the waves $L$ and $T$ radiated by the transducer (Figure 1 ). The four waves reflected back at the upper interface have amplitudes $p_{\eta \mu}$. In the next step, eight waves with the amplitudes $p_{\eta \mu}$, propagate in the upward direction.

The incidence angles of the primary waves $p_{\eta}$ on the upper surface are equal to the inclination angle $\alpha$ (Figure 2). Incidence angles $\gamma_{\eta \mu}$ and $\theta_{\eta \mu \xi}$ of reflected waves are:

$$
\gamma_{\eta \mu}=\beta_{\eta \mu}+\alpha \cdot \theta_{\eta \mu \xi}=\delta_{\eta \mu \xi}+\alpha
$$

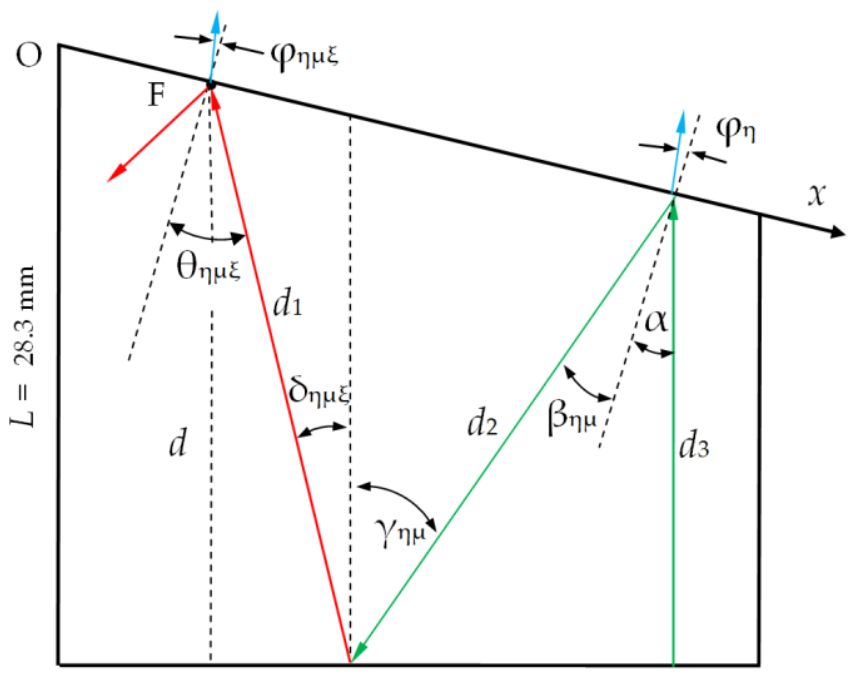

Figure 2. Ray model of the acoustic wave propagation in the AO cell.

The angles of reflection satisfy Snell's law:

$$
\sin \left(\beta_{\eta \mu}\right)=\frac{C_{\mu}}{C_{\eta}} \sin (\alpha), \sin \left(\delta_{\eta \mu \xi}\right)=\frac{C_{\xi}}{C \mu} \sin \left(\gamma_{\eta \mu}\right),
$$

where $C_{L}=5960 \mathrm{~m} / \mathrm{s}$ and $C_{T}=3760 \mathrm{~m} / \mathrm{s}$ are velocities of the longitudinal and transverse waves in fused quartz, respectively [27]. Refracted waves in water propagate at angles $\varphi_{\eta}$ and $\varphi_{\eta \mu \xi}$ :

$$
\sin \left(\varphi_{\eta}\right)=\frac{C_{W}}{C_{\eta}} \sin (\alpha), \sin \left(\varphi_{\eta \mu \xi}\right)=\frac{C_{W}}{C_{\xi}} \sin \left(\theta_{\eta \mu \xi}\right),
$$

where $C_{W}=1485 \mathrm{~m} / \mathrm{s}$ is sound velocity in water.

The spatio-temporal signals received by the focused transducer may be described as

$$
S_{\eta}(x, t)=a_{\eta} w\left(t-t_{\eta}(x)\right), S_{\eta \mu \xi}(x, t)=a_{\eta \mu \xi} w\left(t-t_{\eta \mu \xi}(x)\right),
$$

where $x$ and $t$ are the scanning coordinate and time, $w(t)$ is the impulse response of the experimental setup, $a_{\eta}$ and $a_{\eta \mu \xi}$ are the amplitudes of the compression waves in water generated by corresponding modes $p_{\eta}$ and $p_{\eta \mu \xi}$, and $t_{\eta}$ and $t_{\eta \mu \xi}$ are their delays. The amplitudes $a_{\eta}$ and $a_{\eta \mu \xi}$ are proportional to the amplitudes of the waves penetrated into the immersion liquid:

$$
a_{\eta}=p_{\eta} T_{\eta}(\alpha) h,
$$

where $T_{\eta}(\alpha)$ are the transmission coefficients at the solid-liquid interface and $h$ is the detector sensitivity coefficient. For $p_{L}=1$, this coefficient is equal to

$$
h=\frac{a_{L}}{T_{L}(\alpha)} .
$$


Then, the relative amplitude of the transverse wave $T$ can be found using measured values of $a_{L}$ and $a_{T}$ as

$$
p_{T}=\frac{a_{T} T_{L}(\alpha)}{a_{L} T_{T}(\alpha)}
$$

The amplitudes of the reflected waves may be found as follows:

$$
p_{\eta \mu}=p_{\eta} R_{\eta \mu}(\alpha)
$$

where the reflection coefficient $R_{\eta \mu}(\alpha)$ is determined by the acoustic properties of fused quartz and water. Substitution of (5) and (6) in Equation (7) gives

$$
p_{\eta \mu}=\frac{a_{\eta} R_{\eta \mu}(\alpha)}{h T_{\eta}(\alpha)}=\frac{a_{\eta} R_{\eta \mu}(\alpha) T_{L}(\alpha)}{a_{L} T_{\eta}(\alpha)} .
$$

Since

$$
a_{\eta \mu \xi}=p_{\eta \mu \xi} T_{\xi}\left(\theta_{\eta \mu \xi}\right) h,
$$

the amplitudes $p_{\eta \mu \xi}$ can be estimated in a similar way using the measured values $a_{\eta \mu \xi}$ :

$$
p_{\eta \mu \xi}=\frac{a_{\eta \mu \xi}}{T_{\xi}\left(\theta_{\eta \mu \xi}\right) h}=\frac{a_{\eta \mu \xi} T_{L}(\alpha)}{a_{L} T_{\xi}\left(\theta_{\eta \mu \xi}\right)} .
$$

After the absorber is attached, the direct measurement of the wave amplitudes is not feasible. Nevertheless, the amplitudes of the primary waves $p_{\eta}$ remain the same, whereas the amplitudes of the reflected waves in the cell covered by absorber $p_{\eta \mu}^{*} p_{\eta \mu \xi}^{*}$ decrease in proportion to the reflection or mode conversion coefficient at the quartzabsorber interface $R_{\eta \mu}^{*}(\alpha)$ :

$$
\begin{aligned}
p_{\eta \mu}^{*} & =p_{\eta \mu} \frac{R_{\eta \mu}^{*}(\alpha)}{R_{\eta \mu}(\alpha)}, \\
p_{\eta \mu \xi}^{*} & =p_{\eta \mu \xi} \frac{R_{\eta \mu}^{*}(\alpha)}{R_{\eta \mu}(\alpha)} .
\end{aligned}
$$

The delays $t_{\eta}$ and $t_{\eta \mu \xi}$ of the signals (4) may be calculated as well as the propagation distances of the corresponding waves inside the AO cell (Figure 2). The distance $d$ between the transducer and the receiving focus point F depends linearly

$$
d=L-x \tan \alpha,
$$

where $x$ is the focus position and $L$ is the length of the left facet. The delays of $L$ and $T$ waves are proportional to the distance $x$ :

$$
t_{\eta}=t_{0 \eta}-\varepsilon_{\eta} x
$$

where

$$
t_{0 \eta}=\frac{L}{C_{\eta}}, \varepsilon_{\eta}=\frac{\tan \alpha}{C_{\eta}} .
$$

The delays $t_{\eta \mu \xi}$, depend on the travel distances $d_{1}, d_{2}$ and $d_{3}$ (Figure 2 ) in the following way:

$$
t_{\eta \mu \xi}=\frac{d_{1}}{C_{\xi}}+\frac{d_{2}}{C_{\mu}}+\frac{d_{3}}{C_{\eta}} .
$$

Since

$$
\begin{gathered}
d_{1}=\frac{d}{\cos \delta_{\eta \mu \xi}}, d_{3}=d_{2} \cos \gamma_{\eta \mu}, \text { and } d_{2}=d_{1} \frac{\cos \theta_{\eta \mu \xi}}{\cos \beta_{\eta \mu}} \\
t_{\eta \mu \xi}=t_{0 \eta \mu \xi}-\varepsilon_{\eta \mu \xi} x
\end{gathered}
$$


where

we derive

$$
t_{0 \eta \mu \xi}=K L, \varepsilon_{\eta \mu \xi}=K \cdot \tan \alpha,
$$

$$
K=\frac{1}{\cos \delta_{\eta \mu \xi}}\left(\frac{1}{C_{\xi}}+\frac{\cos \theta_{\eta \mu \xi}}{C_{\mu} \cos \beta_{\eta \mu}}+\frac{\cos \theta_{\eta \mu \xi}}{C_{\eta} \cos \beta_{\eta \mu}} \cos \gamma_{\eta \mu}\right) .
$$

Thus, the values of delay $t_{\eta}$ and $t_{\eta \mu \xi}$ are expressed as a linear dependence on the scanning length $x$ with parameters presented in Table 1.

Table 1. Calculated and experimental values of angular, temporal and slowness parameters of

\begin{tabular}{|c|c|c|c|c|c|c|c|c|}
\hline \multirow[t]{2}{*}{ Wave } & \multicolumn{6}{|c|}{ Calculation } & \multicolumn{2}{|c|}{ Experiment } \\
\hline & $\varphi_{\eta}, \underset{\circ}{\varphi_{\eta \mu \xi}}$ & 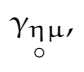 & $\delta_{\eta \mu} \mu$ & $\theta_{\eta \mu} \mu$ & $t_{0 \eta}, t_{0 \eta \mu \xi}, \mu \mathrm{s}$ & $\varepsilon_{\eta}, \varepsilon_{\eta \mu \xi}, \mathrm{ns} / \mathrm{mm}$ & $t_{0 \eta}, t_{0 \eta \mu \xi}, \mu \mathrm{s}$ & $\varepsilon_{\eta}, \varepsilon_{\eta \mu \xi}, \mathrm{ns} / \mathrm{mm}$ \\
\hline$\eta=L$ & 1.62 & - & - & - & 4.755 & 19.1 & 4.75 & 19.3 \\
\hline$\eta=T$ & 2.56 & - & - & - & 7.54 & 30 & 7.9 & 30.8 \\
\hline$\eta \mu \xi=\mathrm{LLL}$ & 4.77 & 13 & 13 & 19.5 & 14.02 & 56.4 & 13.95 & 57.5 \\
\hline$\eta \mu \xi=\mathrm{LLT}$ & 5.74 & 13 & 8.16 & 14.66 & 16.61 & 66.8 & 16.5 & 67.0 \\
\hline$\eta \mu \xi=\mathrm{LTL}$ & 5.70 & 10.6 & 16.9 & 23.5 & 17.2 & 69.2 & 16.8 & 69.1 \\
\hline$\eta \mu \xi=\mathrm{LTT}$ & 6.67 & 10.6 & 10.6 & 17.1 & 19.57 & 78.7 & 19.6 & 80.5 \\
\hline
\end{tabular}
wave propagation.

\section{Experiments}

In this section, we present the experimental results of acoustic pulse detection in two different conditions: (1) an AO cell with absorber fully removed and (2) a similar AO cell partly covered with absorber. We analyze the wave patterns and determine their amplitude and delays in order to estimate $\mathrm{AO}$ cell characteristics.

The measured ultrasonic signals are shown in Figures 3 and 4 as grayscale images. In these images, the signal value is encoded by the gray levels and is presented as a function of the retarded time $\tau$ :

$$
\tau=t_{0}-\varepsilon x
$$

The value of slowness $\varepsilon$ is matched to compensate for the spatial dependence on $\tau$. The $t_{0}$ values were then measured using the positions of the maxima of the ultrasonic pulse envelopes. As the variable part of the propagation time is compensated, the responses $S(x, \tau)$ look like horizontally oriented patterns. The measured values $t_{0}$ and $\varepsilon$ are also presented in Table 1. There is a good agreement between the experimental and calculated values that confirms the correctness of the theoretical model.

The response $S_{L}(x, \tau)$ (Figure 3a) is generated by the main longitudinal wave $L$. There are two horizontal components, $\mathrm{P}_{1}$ and $\mathrm{P}_{2}$, and a set of wavelets $\mathrm{E}$ adjacent to $\mathrm{P}$. The size and position of the components $\mathrm{P}_{1}$ and $\mathrm{P}_{2}$ correspond to the size and position of the sections of the transmitting transducer indicated by $\operatorname{Tr}_{1}$ and $\operatorname{Tr}_{2}$. The wavelets $\mathrm{E}$ are radiated by the transducer's edges. This wave pattern is typical for a flat piston transducer [30].

The values of arrival time $t_{0 T}$ and coefficient $\varepsilon_{T}$ of the transverse wave $T$ are greater than those for the longitudinal wave $L\left(t_{0 L}\right.$ and $\left.\varepsilon_{L}\right)$, since $C_{T}<C_{L}$. The response $S_{T}(x, \tau)$ from wave $T$ also contains two components, $\mathrm{P}_{1}$ and $\mathrm{P}_{2}$, the sizes and positions of which coincide with the transmitting transducer aperture (Figure $3 \mathrm{~b}$ ). Wave $T$ is much weaker than wave $L$ and, therefore, random noise and artifacts (marked by A in Figure $3 b$ ) generated by some unwanted echoes inside the measurement setup are present in the image. 


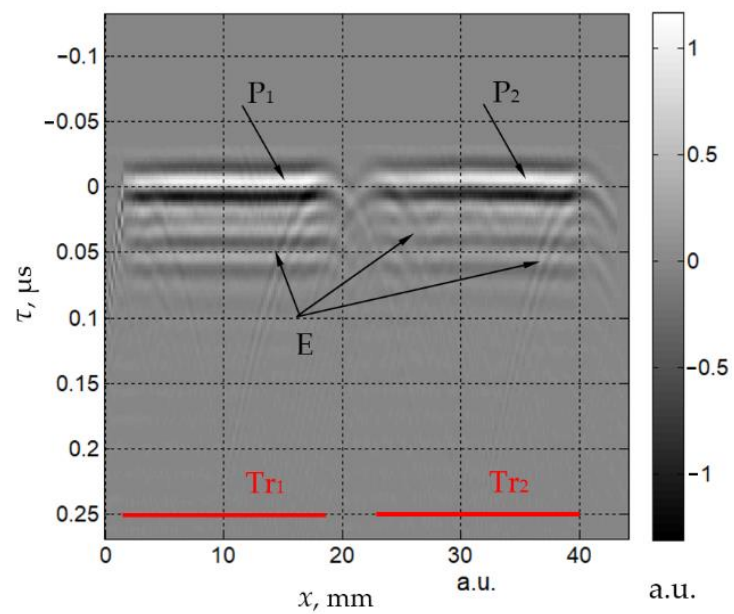

(a)

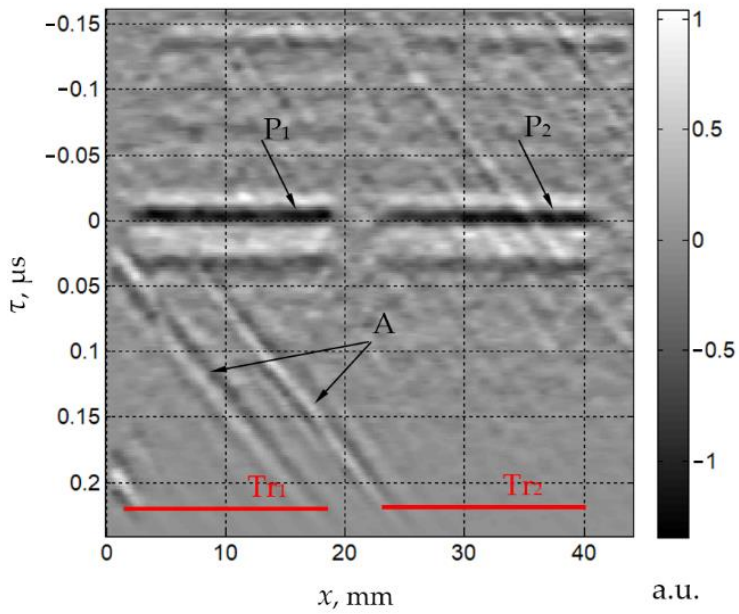

(b)

Figure 3. Measured spatio-temporal signals $S_{L}(x, \tau)(\mathbf{a})$ and $S_{T}(x, \tau)(\mathbf{b})$.

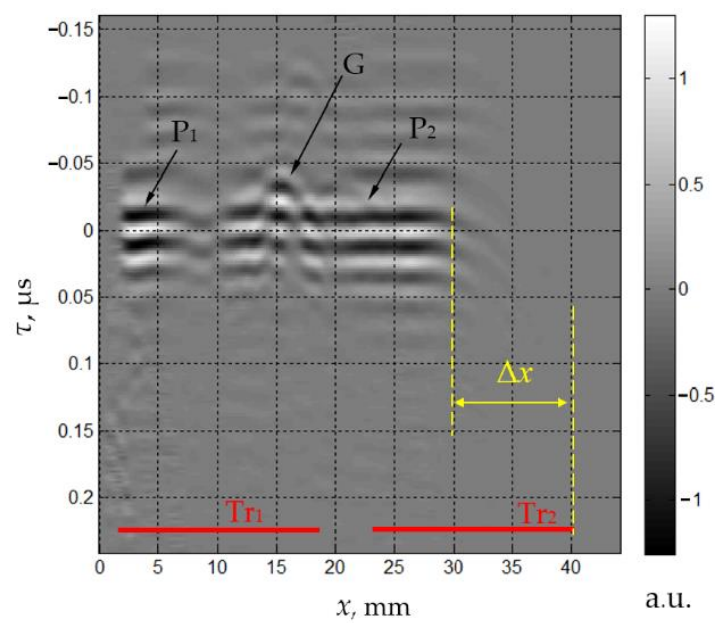

(a)

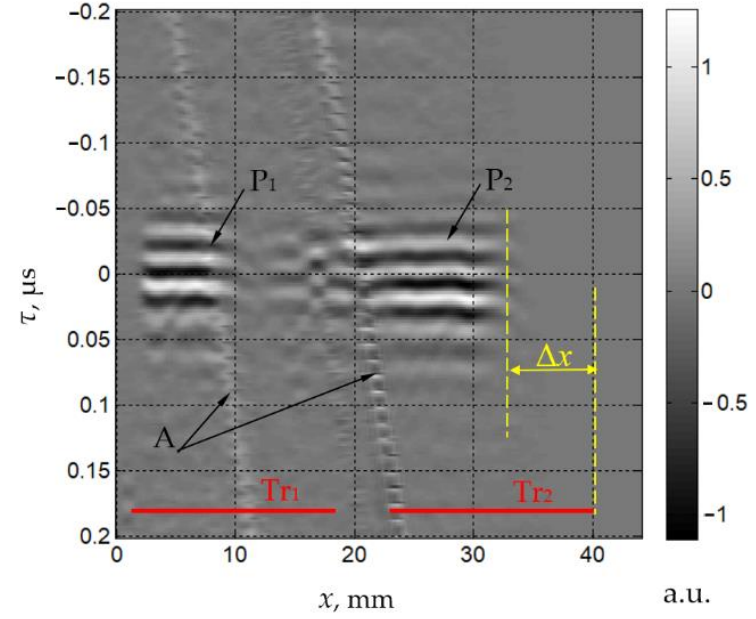

(b)

Figure 4. Measured spatio-temporal signals $S_{L L L}(x, \tau)(\mathbf{a})$ and $S_{L T T}(x, \tau)(\mathbf{b})$.

The LLL wave formed by triple passes of the longitudinal wave is shown in Figure 4a. There is a lateral displacement $\Delta x$ of the response due to the slope of the upper facet. The value of $\Delta x$ may be estimated from the ray model (Figure 2):

$$
\Delta x \approx L\left(\tan \delta_{\eta \mu \xi}+\tan \gamma_{\eta \mu}\right)
$$

The experimentally evaluated displacement is consistent with the theoretical estimate $\Delta x \approx 12 \mathrm{~mm}$. The component $\mathrm{P}_{1}$ is short because the left part of the ultrasonic beam from the section $\operatorname{Tr}_{1}$ is bounded by the left facet of the AO cell. In the wavelet $\mathrm{P}_{2}$, there is irregularity $\mathrm{G}$ formed by the reflection of the wave LL from the gap between two sections of the transducer on the bottom face instead of the reflection from the transducer's surface.

The measured spatio-temporal signal $S_{L T T}(x, \tau)$ is produced by the mode conversion of the longitudinal wave $L$ at the upper interface (Figure $4 \mathrm{~b}$ ). The structure of this response is similar to $S_{L L L}(x, \tau)$, but the displacement $\Delta x \approx 9.5 \mathrm{~mm}$ is less due to the fact that the angles $\delta_{\text {LTT }}$ and $\gamma_{\text {LTT }}$ are less than $\delta_{\text {LLL }}$ and $\gamma_{\text {LLL }}$, respectively (Table 1 ).

To determine the amplitudes of the recorded signals, we estimated the maximal values of the envelopes of the ultrasonic pulses. Due to the irregularity of the transducer radiation efficiency, diffraction effects and artifacts, some spatial variations are present in the received 
responses. To reduce the measurement uncertainty, we calculated the mean and standard deviation of the envelope maximal values within a certain spatial window. The window is set in the range of $30<x<40 \mathrm{~mm}$ for $L$ and $T$ waves (Figure 3). For the rest of the waves (Figure 4), a window of $18<x<28 \mathrm{~mm}$ was used to compensate for the spatial displacement $\Delta x$. The average amplitudes $a_{\eta}$ and $a_{\eta \mu \xi}$ are normalized by the amplitude $a_{L}$. Their relative standard deviations $\sigma_{\eta}$ and $\sigma_{\eta \mu \xi}$ are presented in Table 2. To assess the measurement error of the experimental setup, the random noise level is estimated as the root mean square of the recorded signal in the areas without wave responses and artifacts. This noise value is $0.3 \%$, which is much less than the standard deviations of the amplitudes.

Table 2. Experimental data for absorber-free AO cell and AO cell covered with ultrasound absorber: refracted angles $(\phi)$, normalized amplitudes $(a, p)$, transmission $(T)$ and reflection $(R)$ coefficients for various modes and given inclination angle $\left(\alpha=6.5^{\circ}\right)$.

\begin{tabular}{|c|c|c|c|c|c|c|c|c|}
\hline \multirow{3}{*}{$\begin{array}{l}\text { Wave } \\
\text { Mode }\end{array}$} & \multicolumn{6}{|c|}{$\begin{array}{l}\text { Quartz-Water Interface } \\
\text { (Absorber-Free AO Cell) }\end{array}$} & \multicolumn{2}{|c|}{$\begin{array}{l}\text { Quartz-Epoxy Interface } \\
\text { (Absorber-Covered Cell) }\end{array}$} \\
\hline & \multicolumn{2}{|c|}{ Measured } & \multirow{2}{*}{$\begin{array}{c}\varphi_{\eta}, \\
\varphi_{\eta \mu \xi}\end{array}$} & \multirow[b]{2}{*}{$\begin{array}{c}T_{\eta}(\alpha) \\
T_{\xi}\left(\theta_{\eta \mu \xi}\right)\end{array}$} & \multirow[b]{2}{*}{$R_{\eta \mu}(\alpha)$} & \multirow[b]{2}{*}{$\begin{array}{c}p_{\eta}, p_{\eta \mu} \\
p_{\eta \mu \xi}\end{array}$} & \multirow[b]{2}{*}{$R_{\eta \mu}^{*}(\alpha)$} & \multirow{2}{*}{$\begin{array}{c}p_{\eta}^{*}, p_{\eta \mu,}^{*} \\
p_{\eta \mu \xi}^{*}\end{array}$} \\
\hline & $a_{\eta}, a_{\eta \mu \xi}$ & $\begin{array}{c}\sigma_{\eta}, \\
\sigma_{\eta \mu \xi,} \%\end{array}$ & & & & & & \\
\hline$\eta=L$ & 1 & 8.2 & 1.62 & 0.444 & - & 1 & - & 1 \\
\hline$\eta=T$ & 0.018 & 13.5 & 2.56 & 0.101 & - & 0.078 & - & 0.078 \\
\hline$\eta \mu=\mathrm{LL}$ & - & - & - & - & 0.773 & 0.77 & 0.605 & 0.605 \\
\hline$\eta \mu=\mathrm{LT}$ & - & - & - & - & 0.161 & 0.16 & 0.11 & 0.11 \\
\hline$\eta \mu=\mathrm{TL}$ & - & - & - & - & 0.401 & 0.031 & 0.27 & 0.021 \\
\hline$\eta \mu=\mathrm{TT}$ & - & - & - & - & 0.94 & 0.073 & 0.69 & 0.054 \\
\hline$\eta \mu \xi=\mathrm{LLL}$ & 0.39 & 12.5 & 4.77 & 0.413 & - & 0.42 & - & 0.33 \\
\hline$\eta \mu \xi=\mathrm{LLT}$ & 0.051 & 25 & 5.74 & 0.222 & - & 0.10 & - & 0.068 \\
\hline$\eta \mu \xi=\mathrm{LTL}$ & 0.074 & 22 & 5.70 & 0.398 & - & 0.083 & - & 0.056 \\
\hline$\eta \mu \xi=\mathrm{LTT}$ & 0.075 & 22.6 & 6.67 & 0.257 & - & 0.13 & - & 0.095 \\
\hline
\end{tabular}

To evaluate the influence of the absorber, we used another AO cell of the same design. The cell has the absorber installed on the upper facet. The absorber is made from epoxy resin and covers a part of the facet as shown in Figure 5. The left edge of the absorber is located at $x_{0} \approx 30 \mathrm{~mm}$. Therefore, the signal $S_{L L L}(x, \tau)$ is damped at the interval $\left[x_{1}, x_{2}\right]$ $\approx[18,28] \mathrm{mm}$ due to the decrease of the reflection coefficient $R_{L}$ in the presence of the absorber. The ratio of amplitudes measured for the quartz-absorber and quartz-water configurations is 0.75 .

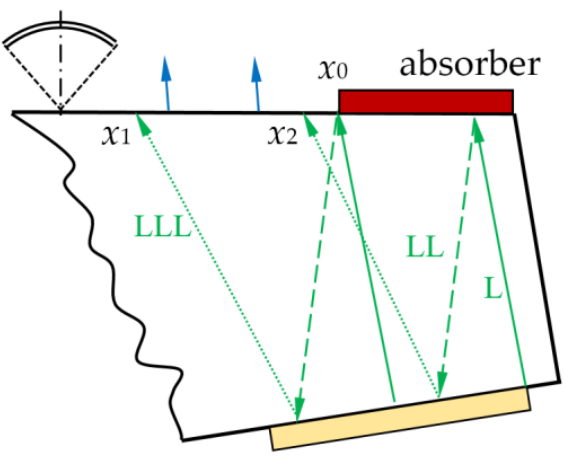

Figure 5. Ray propagation in $\mathrm{AO}$ cell with absorber. 


\section{Discussion}

The wave amplitudes in quartz $p_{T}, p_{\eta \mu}$ and $p_{L \mu \xi}$ are determined from the ones measured in water $a_{\eta}$ and $a_{\eta \mu \xi}$ (Table 2) using Equations (7), (9) and (11). Well-known formulas [29] allows calculation of the transmission coefficients $T_{\eta}(\alpha)$ and $T_{\xi}\left(\theta_{\eta \mu \xi}\right)$, reflection and mode conversion coefficients $R_{\eta \mu}(\alpha)$ for the fused quartz-water interface. These coefficients are shown in Figure 6 as functions of the refraction angle $\varphi$ in water. Their values at the angles $\varphi_{\eta}, \varphi_{\eta \mu \xi}$ are presented in Table 2 as well as the obtained relative amplitudes $p_{\eta}, p_{\eta \mu}$ and $p_{\eta \mu \xi}$.

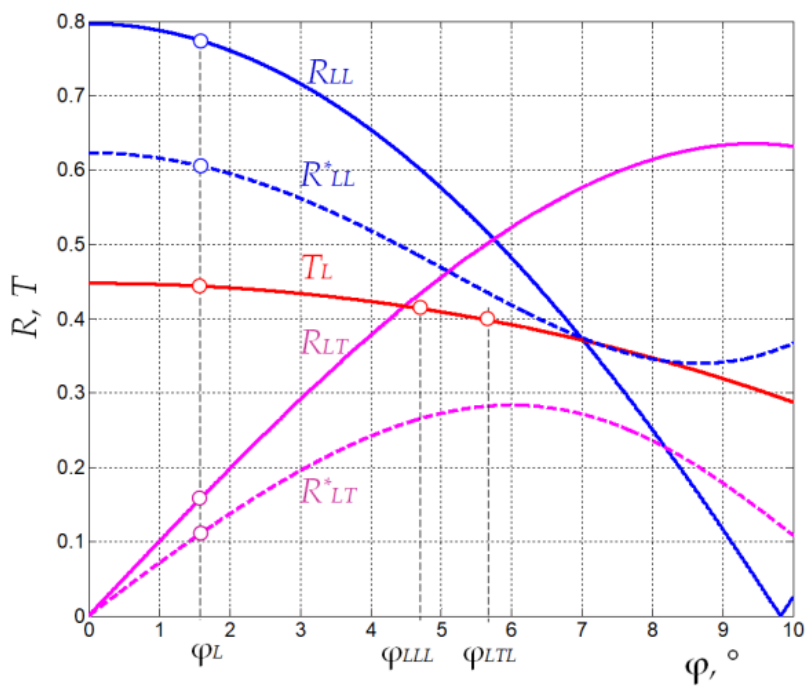

(a)



(b)

Figure 6. (a) Reflection coefficients $R_{L L}, R_{L L}^{*}, R_{T T}$ and $R_{T T}^{*}$, transmission coefficients $T_{L}$, and $T_{T}$, mode conversion coefficients $R_{L T}, R_{L T}^{*}, R_{T L}$ and $R_{T L}^{*}$ for the interfaces fused quartz-water (solid lines) and fused quartz-absorber (dashed lines) vs refraction angle in water $\varphi$ : longitudinal (a) and transverse (b) waves in quartz.

We have discovered that the calculated relative amplitude of the direct transverse wave $p_{T}=0.078$ is significant. Thus, the ultrasonic transducer generates a non-negligible transverse wave along with the regular longitudinal wave. This unwanted wave propagates in the same direction and cannot be attenuated by the absorber.

Among the reflected waves, the LL wave with an amplitude of $p_{L L}=0.77$ is the largest. The waves TL and TT, generated at the upper interface by the mode conversion of the wave $L$ and reflection of the transverse wave $T$, are much weaker. Therefore, only the propagation of LL and LT waves is considered below.

We should note that the amplitudes $p_{\eta \mu \xi}$ may be calculated using the determined values $p_{\eta \mu}$ and the reflection coefficients at the bottom interface. However, the design of the transmitting transducer is rather complicated and it is difficult to find the reflection coefficients from its surface. In addition, the reflectivity depends on the electrical load of the transducer. Thus, this approach is not reliable and barely practically realizable.

In the presence of the absorber, the amplitudes $p^{*}{ }_{\eta}$ of the direct waves do not change, while the amplitudes $p^{*} \eta \mu$ and $p^{*} \eta \mu \xi$ become smaller. These amplitudes are estimated from the amplitudes $p_{\eta \mu}$ and $p_{\eta \mu \xi}$ via Equations (12) and (13) (Table 2). We calculated the required reflection and mode conversion coefficients $R^{*}{ }_{\eta \mu}(\alpha)$ under the assumption that the absorber is a solid medium with a density of $1150 \mathrm{~kg} / \mathrm{m}^{3}$ and velocities of longitudinal and transvers waves of $2650 \mathrm{~m} / \mathrm{s}$ and $1100 \mathrm{~m} / \mathrm{s}$, respectively, values which are typical for epoxy resin [31]. The coefficients $R_{\eta \mu}^{*}$ presented in Figure 6 and Table 2 are obtained based on well-established technique [29]. In the experimental data, the ratio $p^{*}{ }_{\mathrm{LLL}} / p_{\mathrm{LLL}}$ is 0.75 , which is quite close to the value 0.79 calculated from Table 2 . We tested the proposed method 
on a model absorber and confirmed its applicability. Though the estimated amplitudes $p_{\eta \mu}^{*}$ and $p_{\eta \mu \xi}^{*}$ are small, they are not negligible and their effect may be significant for the detailed analysis of the $\mathrm{AO}$ device characteristics.

\section{Conclusions}

We have shown that impulse acoustic microscopy is quite informative tool for quantitative characterization of the acoustic field in $\mathrm{AO}$ cells. It allows to define the modes, amplitudes, propagation trajectories and other features of the ultrasonic waves propagating in the crystal even after multiple reflections. This information is highly important in practice as it enables the evaluation of the correctness of AO cell design including the cut and facet angles, efficiency of the ultrasound piezotransducer and absorber functioning, etc. Being non-destructive and highly sensitive, impulse acoustic microscopy might be effective for quite fast experimental validation of theoretical estimations and numerical modeling results as well as for accurate certification of $\mathrm{AO}$ devices.

Author Contributions: Conceptualization, S.A.T. and V.E.P.; methodology, S.A.T.; validation, V.E.P. and A.S.M.; formal analysis, V.E.P.; resources, S.A.T. and A.S.M.; data curation, S.A.T.; writingoriginal draft preparation, S.A.T. and A.S.M.; writing-review and editing, V.E.P.; project administration, A.S.M. All authors have read and agreed to the published version of the manuscript.

Funding: This research was funded by Federal State Task Program of Scientific and Technological Center of Unique Instrumentation RAS. This work was performed using the equipment of the Shared Research Facilities of the Scientific and Technological Centre of Unique Instrumentation of the Russian Academy of Sciences.

Institutional Review Board Statement: Not applicable.

Informed Consent Statement: Not applicable.

Data Availability Statement: The data presented in this study are available on request from the corresponding author.

Conflicts of Interest: The authors declare no conflict of interest.

\section{References}

1. Goutzoulis, A.P.; Rape, D.R. Design and Fabrication of Acousto-Optic Devices; CRC Press: Boca Raton, FL, USA, 2004; 520p.

2. Xu, J.; Stroud, R. Acousto-Optic Devices: Principles, Design, and Applications; Wiley: New York, NY, USA, 1992; 672p.

3. Römer, G.R.B.E.; Bechtold, P. Electro-optic and Acousto-optic Laser Beam Scanners. Phys. Procedia 2014, 56, 29-39. [CrossRef]

4. Hagen, N.; Kudenov, M.W. Review of Snapshot Spectral Imaging Technologies. Opt. Eng. 2013, 52, 090901. [CrossRef]

5. Duocastella, M.; Surdo, S.; Zunino, A.; Diaspro, A.; Saggau, P. Acousto-optic systems for advanced microscopy. J. Phys. Photonics 2020, 3, 012004. [CrossRef]

6. Voloshinov, V.; Polikarpova, N. Acousto-optic investigation of propagation and reflection of acoustic waves in paratellurite crystal. Appl. Opt. 2009, 48, 55-66. [CrossRef] [PubMed]

7. Maak, P.; Takács, T.; Barocsi, A.; Kollar, E.; Richter, P. Thermal behavior of acousto-optic devices: Effects of ultrasound absorption and transducer losses. Ultrasonics 2011, 51, 441-451. [CrossRef] [PubMed]

8. Balakshy, V.; Voloshin, A.; Molchanov, V. Anisotropic light diffraction in crystals with a large acoustic-energy walk-off. Opt. Spectrosc. 2014, 117, 801-806. [CrossRef]

9. Voloshinov, V.; Polikarpova, N.; Ivanova, P.; Khorkin, V. Acousto-optic control of internal acoustic reflection in tellurium dioxide crystal in case of strong elastic energy walkoff [Invited]. Appl. Opt. 2018, 57, C19-C25. [CrossRef]

10. Kudo, N. Optical methods for visualization of ultrasound fields. Jpn. J. Appl. Phys. 2015, 54, 07HA01. [CrossRef]

11. Ishikawa, K.; Yatabe, K.; Oikawa, Y. Physical-model-based reconstruction of axisymmetric three-dimensional sound field from optical interferometric measurement. Meas. Sci. Technol. 2021, 32, 045202. [CrossRef]

12. Bertling, K.; Perchoux, J.; Taimre, T.; Malkin, R.; Robert, D.; Rakić, A.D.; Bosch, T. Imaging of acoustic fields using optical feedback interferometry. Opt. Express 2014, 22, 30346-30356. [CrossRef]

13. Rajput, S.K.; Matoba, O.; Takase, Y.; Inoue, T.; Itaya, K.; Kumar, M.; Quan, X.; Xia, P.; Awatsuji, Y. Multimodal sound field imaging using digital holography [Invited]. Appl. Opt. 2021, 60, B49-B58. [CrossRef] [PubMed]

14. Malkin, R.; Todd, T.; Robert, D. A simple method for quantitative imaging of 2D acoustic fields using refracto-vibrometry. J. Sound Vib. 2014, 333, 4473-4482. [CrossRef]

15. Roberto, L.; Vanlanduit, S.; Arroud, G.; Guillaume, P. Underwater Acoustic Wavefront Visualization by Scanning Laser Doppler Vibrometer for the Characterization of Focused Ultrasonic Transducers. Sensors 2015, 15, 19925-19936. [CrossRef] 
16. Ortiz, P.F.U.; Perchoux, J.; Arriaga, A.L.; Jayat, F.; Bosch, T. Visualization of an acoustic stationary wave by optical feedback interferometry. Opt. Eng. 2018, 57, 051502. [CrossRef]

17. Wu, J.; Xu, Z.; Li, K.; Lv, G.; Li, X.; Wang, C. Analysis of Acoustic Near Field Characteristics in Acousto-Optic Modulator. IEEE Photonics Technol. Lett. 2021, 33, 201-204. [CrossRef]

18. Yatabe, K.; Ishikawa, K.; Oikawa, Y. Acousto-optic back-projection: Physical-model-based sound field reconstruction from optical projections. J. Sound Vib. 2017, 394, 171-184. [CrossRef]

19. Torras-Rosell, A.; Barrera-Figueroa, S.; Jacobsen, F. Sound field reconstruction using acousto-optic tomography. J. Acoust. Soc. Am. 2012, 131, 3786-3793. [CrossRef] [PubMed]

20. Verburg, S.A.; Fernandez-Grande, E. Acousto-Optical Volumetric Sensing of Acoustic Fields. Phys. Rev. Appl. 2021, 16, 044033. [CrossRef]

21. Koponen, E.; Leskinen, J.; Tarvainen, T.; Pulkkinen, A. Acoustic pressure field estimation methods for synthetic schlieren tomography. JASA 2019, 145, 2470-2479. [CrossRef]

22. Xu, Z.; Chen, H.; Yan, X.; Qian, M.-L.; Cheng, Q. Three-dimensional reconstruction of nonplanar ultrasound fields using Radon transform and the schlieren imaging method. JASA 2017, 142, EL82-EL88. [CrossRef]

23. Pulkkinen, A.; Leskinen, J.; Tiihonen, A. Ultrasound field characterization using synthetic schlieren tomography. JASA 2017, 141, 4600-4609. [CrossRef] [PubMed]

24. Scruby, C.B.; Drain, L.E. Laser Ultrasonics Techniques and Applications, 1st ed.; Taylor \& Francis Group: New York, NY, USA, 1990; 462p.

25. Hill, C.R.; Bamber, J.C.; ter Haar, G.R. Physical Principles of Medical Ultrasonics, 2nd ed.; John Wiley \& Sons: West Sussex, UK, 2004; 528p.

26. Harris, G.R. Hydrophone measurements in diagnostic ultrasound fields. IEEE Trans. Ultrason. Ferroelect. Freq. Contr. 1988, 35, 87-101. [CrossRef] [PubMed]

27. Briggs, G.A.D.; Kolosov, O.V. Acoustic Microscopy, 2nd ed.; Oxford university press: New York, NY, USA, 2010; 387p.

28. Vogt, M.; Ermert, H. High-frequency ultrasonic systems for high-resolution ranging and imaging. In Advances in Acoustic Microscopy and High Resolution Ultrasonic Imaging: From Principles to New Applications; Maev, R.G., Ed.; Wiley \& Son-VCH Verlag \& Co, KGaA: Weinheim, Germany, 2013; pp. 93-123.

29. Brekhovshikh, L.M.; Godin, O.A. Acousics of Layered Media I; Springer: Heidelberg, Germany, 1990; pp. 87-108.

30. Kino, G.S. Acoustic Waves: Devices, Imaging and Analog Signal Processing; Prentice-Hall Inc.: Englewood Cliffs, NJ, USA, 1987; pp. 164-218.

31. Birks, A.S.; Green, R.E.; McIntire, P. Nondestructive Testing Handbook, 2nd ed.; The American Society For Nondestructive Testing: Columbus, OH, USA, 1991; Volume 7, pp. 836-841. 